\title{
Lipoprotein Candidate Genes for Multivariate Factors of the Insulin Resistance Syndrome: A Sib-pair Linkage Analysis in Women Twins
}

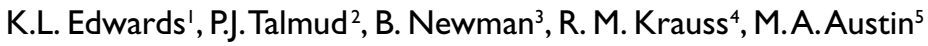 \\ I Department of Epidemiology, School of Public Health and Community Medicine, University of Washington, Seattle, WA \\ 2 Division of Cardiovascular Genetics, Department of Medicine, Rayne Institute, University College London, London \\ 3 School of Public Health, Queensland University of Technology, Brisbane, Queensland, Australia \\ 4 Department of Molecular and Nuclear Medicine, Life Sciences Division, Ernest Orlando Lawrence Berkeley National Laboratory, Berkeley, CA \\ 5 Institute for Public Health Genetics, School of Public Health and Community Medicine, University of Washington, Seattle, WA
}

\begin{abstract}
The insulin resistance syndrome (IRS) is characterized by a combination of interrelated coronary heart disease risk factors, including low high-density lipoprotein cholesterol (HDLC) levels, obesity and increases in triglyceride (TG), systolic and diastolic blood pressure (BP), small low-density lipoprotein particles (LDL-size), and fasting and postload plasma insulin and glucose. Using factor analysis, we previously identified multivariate factors based on data from women participating in the Kaiser Permanente Women Twins Study: 1) Weight/Fat, 2) Insulin/Glucose, 3) Lipids, and 4) BP. The purpose of this study is to evaluate evidence for genetic linkage between the multivariate factors and candidate genes. Quantitative sib-pair analysis based on the factor scores with markers for 9 candidate genes was carried out based on data from 126 pairs of dizygotic (DZ) women twins from the second exam of the Kaiser Permanente Women Twins study. Suggestive evidence for linkage was found for the Weight/fat factor and the Apo $E$ gene $(p=0.01)$, and stronger evidence for linkage with the Lipid factor and the cholesterol ester transfer protein ( $p=$ 0.002) gene. Therefore, the CETP gene appears to influence covariation in LDL size, TG, and HDL, and may account for a portion of the well-established statistical and metabolic associations observed between these risk factors.
\end{abstract}

The insulin resistance syndrome (IRS) (DeFronzo and Ferrannini, 1991), also known as Syndrome X (Reaven, 1988), is characterized by a clustering of type 2 diabetes and coronary heart disease (CHD) risk factors, including hypertension, dyslipidemia, glucose intolerance, and central obesity (DeFronzo and Ferrannini, 1991; Ferrannini et al., 1991; Modan et al., 1985; Reaven, 1988). Although genetic influences on individual features of the IRS are well established (Bouchard and Perusse, 1993; Liese et al., 1998), the metabolic and possible genetic basis for the clustering of risk factors remains to be elucidated.

A variety of statistical approaches provide evidence for underlying genetic influences on the interrelated features of the IRS, including body mass index (BMI) and waist-tohip ratio (WHR) (Cardon et al., 1994) and the clustering of hypertension, diabetes and obesity (Carmelli et al.,
1994). Using quantitative genetic analysis, several studies suggest common additive genetic influences (pleiotropy) on pairs of risk factors characterizing the IRS, including high density lipoproprotein (HDL) cholestrol and triglyceride (TG), BMI and fat mass (Mahaney et al., 1995), and insulin levels and BMI, WHR, subscapular skinfolds and HDL cholesterol (Mitchell et al., 1996; Rainwater et al., 1997). A recent, trivariate, quantitative genetic analysis in hypertriglyceridemic families also reported strong additive genetic correlations between each pair of lipid and lipoprotein traits, including low-density lipoprotein (LDL) size, TG and HDL (Edwards et al., 1999). Despite this accumulating evidence there have been no reports of specific genes that may underlie these multivariate traits characterizing the syndrome.

Hence, the purpose of this study is to evaluate evidence for genetic linkage, using a sib-pair approach, between previously identified multivariate factors characteristic of the IRS (Edwards et al., 1994), and 9 candidate genes involved in lipid and lipoprotein metabolism, using data from a sample of dizygotic women twins.

\section{$\overline{\text { Method }}$}

\section{Study Subjects}

Study subjects were participants in the second examination of the Kaiser Permanente Women Twins Study in Oakland, California. Examination 2 was conducted between 1989 and 1990, and included 704 individuals (206 monozygotic (MZ) and 146 dizygotic (DZ) pairs), representing $81 \%$ of the original cohort examined in 1979-1980 (Austin et al., 1987; Selby et al., 1993). Zygosity was determined at

Address for Correspondence: Karen L. Edwards, Ph.D., Department of Epidemiology, Box 357236, School of Public Health and Community Medicine, University of Washington, Seattle, WA 98195, USA.Email: keddy@u.washington.edu

Received: 4 December, 2000, accepted 8 January, 2001 
examination 1 based on 20 polymorphic loci, such that the probability of misclassification of a pair who were concordant on all markers as monozygotic was less than 0.001 (Austin et al., 1987). At the time of the second exam, each woman completed a health history questionnaire and a physical examination, including anthropometric and laboratory measurements. The average age of individual women at examination 2 was 51 years, and the majority (90\%) of women in the sample were white.

\section{Data Collection}

The data for this analysis has previously been described in detail (Edwards et al., 1994). Briefly, weight was measured without shoes and in lightweight clothing. Waist circumference was measured using a standardized protocol. Systolic and diastolic blood pressure was measured after the subject had been seated for five minutes using a mercury sphygmomanometer. Plasma glucose and insulin were both measured following an overnight fast, and again two hours after a $75 \mathrm{~g}$ oral glucose load (Glutol, Paddock Laboratories, Minneapolis, MN) as previously described (Edwards et al., 1994). After the overnight fast, $30 \mathrm{ml}$ of whole blood was collected into EDTA-containing tubes for lipid determinations. Plasma was separated by centrifugation within two hours, and stored under refrigeration. Nondenaturing gradient gel electrophoresis was performed on the plasma using 2-16\% polyacrylamide gradient gels (Pharmacia) (Nichols et al., 1986). The estimated diameter of the major LDL subclass was calculated based on a calibration curve constructed from high molecular weight standards run on the same gel (Krauss and Burke, 1982). The diameter, denoted LDL peak particle diameter (LDL-PPD), is a continuous variable and is used in the factor analysis as a measure of LDL heterogeneity. Total HDL cholesterol (HDL-C) (Warnick et al., 1985) and triglycerides (Nagele et al., 1984) were determined by standardized methods at the Donner Laboratory (Berkeley, CA).

\section{Candidate Genes}

Markers were genotyped for each of the following 9 candidate genes as previously described (Austin et al., 1998; Friedlander et al., 2000; Talmud et al., 2000): apolipopro- tein E (apoE), apolipoprotein B (apoB), apolipoprotein CIII (apo CIII), the low density lipoprotein receptor (LDL-R), hormone sensitive lipase (HSL), microsomal triglyceride transfer protein (MTP), hepatic lipase (HL), the insulin receptor (IR), and cholesterol ester transfer protein (CETP). The most highly polymorphic markers available were selected, with the number of alleles ranging from 3 (apoE) to 28 (CETP) (Table 1). Briefly, genotyping of the apoE gene, to identify the 3 alleles $\varepsilon 2$, $\varepsilon 3$, and $\varepsilon 4$, used the method of Bolla et al., (1995). The apoB VNTR in the 3' untranslated region was genotyped according to the method of Boerwinkle et al., (1989). The CETP tetranucleotide $(\mathrm{GAAA})^{\mathrm{n}}$ repeat was recently identified and described by Talmud et al., (2000). All other genotypes were carried out using ABI fluorescent labeled forward primers (synthesized by Oswell Ltd, Southhampton UK; non fluorescent reverse primers were synthesized by GibcoBRL, Paisley, UK).

\section{Statistical Analysis}

Factor analysis. The general approach for this analysis has been described in detail elsewhere (Edwards et al., 1994). Briefly, the analysis included the following characteristics of the IRS: body weight, waist circumference, fasting and postload glucose and insulin, systolic and diastolic blood pressures, TG, HDL-cholesterol, and LDL-PPD. Principal component analysis was first used to extract the new, uncorrelated "components". In concurrence with the literature (Stevens, 1986), components with eigenvalues greater than or equal to 1.0 were retained in the analysis. As previously described, four components were retained and rotated orthogonally using Varimax rotation, and are referred to as factors, each representing a linear combination of the 11 individual variables. However, because this analysis focuses on candidate genes for insulin and lipid and lipoproteins, the Blood Pressure factor (factor 4) was not included in the sib-pair linkage analysis.

A factor score was calculated for each woman as a weighted sum of the values of the standardized variables, using the scoring coefficients as the weights. All variables included in the factor analysis contribute to each of the factor scores. Each woman has three factor scores, one for

Table 1

Candidate Gene Polymorphisms Typed for Women Twins

\begin{tabular}{lclcc}
\hline Candidate Gene & Chromosome & \multicolumn{1}{c}{ Marker } & No. of Alleles & Heterozygosity Index* $^{*}$ \\
\hline ApoE & 19 & Triallelic: Isoforms: $\varepsilon 2, \varepsilon 3$, and $\varepsilon 4$ & 3 & 0.43 \\
ApoB & 2 & VNTR at 3' end & 14 & 0.74 \\
& & Tetranucleotide repeat in intron 20 & 6 & 0.60 \\
Apo CIII & 11 & Tetranucleotide repeat in intron 3 & 7 & 0.60 \\
LDL-R & 19 & D19S394, 250 kb 5' to LDL-R gene & 21 & 0.91 \\
HSL & 19 & GT repeat in intron 7 & 14 & 0.67 \\
MTP & 4 & CA repeat in intron 10 & 13 & 0.74 \\
HL & 15 & CA repeat in intron 8 & 7 & 0.63 \\
IR & 19 & CT repeat in intron 2 & 12 & 0.51 \\
CETP & 16 & GAAA repeat in promotor region & 28 & 0.73 \\
\hline
\end{tabular}

* Based on all individual women typed in the study. (Adapted from Austin et al., 1998) 
each factor of interest, that were used as continuous variables in the sib-pair linkage analysis.

Sib Pair Analysis. Quantitative sib-pair linkage analysis was used to test for genetic linkage between the factors characterizing the IRS and the candidate genes. The sib-pair method has the advantage of being a nonparametric method of linkage analysis that requires no a priori knowledge of the mode of inheritance of the phenotype under investigation. The rationale underlying this method is that, in the presence of linkage between a marker locus and the quantitative trait, sib pairs having more similar phenotypes will, on average, share a greater proportion of genes (alleles) identical by descent (IBD) at the marker locus. The sib-pair method used here is implemented in the SIBPAL program of the S.A.G.E. package (Release 2.2, 1994). In this regression approach, quantitative variation in the trait (factor scores) is examined in sibships as a function of shared alleles for the polymorphic marker of each candidate gene. A regression line is then calculated with the squared difference in the factor score between sibs (squaring is used to obtain only positive values) as the dependent variable and the estimated number of shared alleles IBD as the independent variable (Haseman \& Elston, 1972). If the slope of this line is negative, and significantly different from zero with the appropriate degrees of freedom (Blackwelder \& Elston, 1982), the result is interpreted as evidence for linkage between the phenotype and the candidate gene. In addition, the slope of the regression line reflects the magnitude of evidence for linkage.

In this sib-pair analysis, because parental genotypes were not available observed allele sharing represents identity by state (IBS). However, by using allele frequency estimates from each marker IBD can be estimated from IBS. This estimation procedure is implemented in the SIBPAL subroutine of S.A.G.E., and is based on an algorithm described by Amos and Elston (1989). Using this procedure the resulting IBD values differ from 0,1 , and 2 .
Finally, because population-based allele frequencies were not available for several of the markers used in this study, all allele frequency estimates for IBD estimation were based on the sample of DZ women twins.

Due to the requirement of intact twin pairs for genetic analysis, pairs were excluded if one or both co-twins were missing data for any variable included in the factor analysis, were diabetic, or if either twin had triglyceride values greater than $400 \mathrm{mg} / \mathrm{dl}$. After exclusions there were a total of 126 pairs of DZ women twins available for analysis. However, the sample size varies slightly for each candidate gene due to missing genotype data. No adjustment for age or gender is needed since all pairs are perfectly matched on these characteristics. Because the analysis includes multiple comparisons, a $p$-value of $<0.01$ was considered statistically significant evidence for linkage.

\section{Results}

The three factors of interest are shown in Table 2 and have been previously described (Edwards et al., 1994). Briefly, factor 1 is characterized by large positive factor loadings for body weight, waist circumference and fasting insulin, and is interpreted as a weight/fat factor. Factor 2 is characterized by large positive factor loadings for fasting insulin, fasting glucose, postload insulin and postload glucose, and is interpreted as an insulin/glucose factor. Factor 3 is characterized primarily by TG, HDL and LDL-PPD and is interpreted as a lipid factor. For the present analysis, the resulting factors are interpreted as composite variables representing multivariate phenotypes of the IRS.

Table 3 presents the sib-pair results for each of the three factors and each of the 9 candidate genes. The strongest evidence for linkage was observed between the lipid factor and the CETP gene (Figure 1). The significantly negative slope indicates that those pairs who are more alike at the CETP marker locus (slope $=-1.94, p=0.002$ ) have more similar scores for the lipid factor. The apoE gene showed modest

\section{Table 2}

Results of Factor Analysis: Factors and Factor Loadings ${ }^{*}$

\begin{tabular}{lccc} 
& & Factors & \\
Variable & 1 & 2 & 3 \\
(Weight/Fat) & (Insulin/Glucose) & (Lipids) \\
\hline Weight & 0.93 & 0.06 & -0.10 \\
Waist & 0.88 & 0.20 & -0.24 \\
F. Ins & 0.60 & 0.49 & -0.18 \\
F. Glu & 0.17 & 0.88 & 0.09 \\
PL. Ins & 0.43 & 0.40 & -0.27 \\
PL. Glu & 0.11 & 0.89 & -0.19 \\
TG & 0.11 & 0.20 & -0.79 \\
HDL & -0.40 & -0.02 & 0.64 \\
LDL-PPD & -0.11 & -0.17 & 0.89 \\
DBP & 0.11 & 0.03 & -0.04 \\
SBP & 0.09 & 0.22 & -0.13
\end{tabular}

*Factor loadings represent the correlation between the individual variable and each factor. Components are rotated using Varimax rotation to obtain factors; $\mathrm{F}$., fasting; $\mathrm{PL}$., postload; TG, triglyceride; HDL, high density lipoprotein cholesterol; and LDL-PPD, low-density lipoprotein peak particle diameter; DBP, diastolic blood pressure; SBP, systolic blood pressure. (Adapted from Edwards et al., 1994) 
Table 3

Slope and P-values from Quantitative Sib Pair Linkage Analysis based on Dizygous Women Twins

\begin{tabular}{|c|c|c|c|c|c|c|c|}
\hline \multirow{2}{*}{$\begin{array}{l}\text { Candidate } \\
\text { Gene Marker } \\
\text { ApoE }\end{array}$} & \multirow{2}{*}{$\begin{array}{c}\text { No. of } \\
\text { Pairs }\end{array}$} & \multicolumn{6}{|c|}{$\begin{array}{l}\text { Regression Slope ( } p \text { value) } \\
\text { Factor }\end{array}$} \\
\hline & & -4.32 & $(0.013)$ & 1.40 & $(0.626)$ & 0.40 & $(0.618)$ \\
\hline ApoB VNTR & 107 & 1.36 & $(0.865)$ & -1.89 & $(0.240)$ & -1.67 & $(0.027)$ \\
\hline ApoB Intron & 107 & 1.58 & $(0.867)$ & 0.53 & $(0.567)$ & -0.05 & (0.479) \\
\hline Apo CIII & 109 & 2.41 & $(0.986)$ & -1.37 & $(0.087)$ & 0.95 & $(0.830)$ \\
\hline LDL-R & 110 & 0.17 & $(0.565)$ & -3.60 & $(0.053)$ & -0.71 & (0.154) \\
\hline HSL & 112 & 0.10 & $(0.532)$ & -0.08 & $(0.487)$ & -1.48 & $(0.031)$ \\
\hline MTP & 108 & 0.25 & $(0.581)$ & -2.50 & $(0.172)$ & -0.50 & $(0.269)$ \\
\hline $\mathrm{HL}$ & 109 & -0.06 & $(0.482)$ & -3.68 & $(0.107)$ & 0.78 & $(0.801)$ \\
\hline IR & 110 & 0.06 & $(0.516)$ & -0.12 & $(0.484)$ & -1.00 & $(0.162)$ \\
\hline CETP & 107 & 0.91 & $(0.949)$ & -3.01 & $(0.081)$ & -1.94 & $(0.002)$ \\
\hline
\end{tabular}

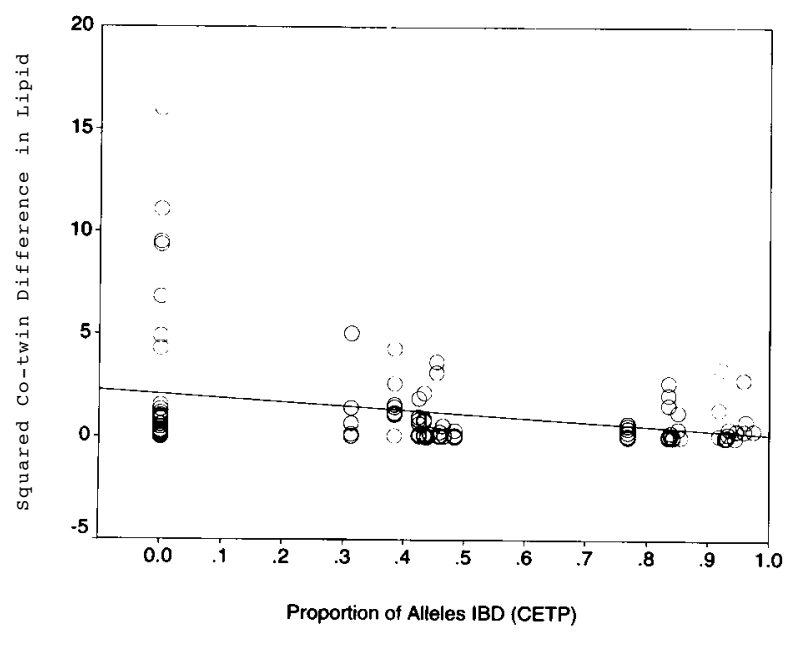

Figure 1

Results of quantitative sib-pair linkage analysis of the CETP gene in DZ women twins. The $\mathrm{X}$-axis indicates the estimated number of alleles shared IBD by co- twins in a pair for the CETP marker (see Table 1). The $\mathrm{Y}$-axis indicates the squared co-twin difference in the Lipid factor. The significant negative slope $(p=0.002)$ of the regression line is consistent with evidence for genetic linkage.

evidence for linkage to the weight/fat factor. The negative slope (slope $=-4.3, p=0.01)$ indicates that those pairs who are more alike at the apoE locus have more similar scores for the weight/fat factor, consistent with linkage.

\section{$\overline{\text { Discussion }}$}

These results represent the first report of linkage between multivariate phenotypes of the IRS, defined by factor analysis, and candidate genes for lipid and lipoprotein metabolism. Specifically, these results are consistent with linkage between the lipid factor and the CETP gene, and possible genetic linkage between the body weight/fat factor and the apoE gene. Importantly, these results extend previous work from quantitative genetic analyses (Mahaney et al., 1995; Mitchell et al., 1996; Edwards et al., 1999) and suggest that single major genes, such as the CETP gene, contribute to the well-established covariation between LDL size, TG and HDL.

The primary finding in this report is the apparent linkage between the lipid factor and the highly polymorphic marker within the CETP gene promoter. This result is consistent with several previous reports based on the individual lipid and lipoproteins characterizing the lipid factor. Rotter et al. reported evidence for linkage with LDL size and an anonymous marker (D16S313) within $6 \mathrm{cM}$ of the CETP gene on chromosome 16 (Rotter et al., 1996). Allayee et al. (1998) recently reported linkage to microsatellite markers flanking the CETP and lecithin:cholesterol acyltransferase (LCAT) genes to small dense LDL in familial combined hyperlipidemic families. Studies have also demonstrated an association between variation in the CETP gene and HDL levels (Gudnason et al., 1999; Kuivenhoven et al., 1998).

Importantly, these results extend the previous report by Talmud et al. showing evidence for linkage between the CETP gene and several individual phenotypes, including LDL size, TG and Apo B levels in the same sample of women twins. Taken together, these results suggest that variation in the CETP gene influences not only LDL size, TG, and possibly HDL and ApoB levels, but also a composite phenotype characterized by these lipids and lipoproteins. Together, these results suggest that the CETP gene could have pleiotropic effects on these risk factors. Adding support to this hypothesis is the work by Comuzzie et al. (1997), who indicates that a synthetic phenotype extracted using principal component analysis is of greater utility in linkage analysis when the majority of pleiotropy is attributable to major loci, rather than polygenes. Thus, the CETP gene may account for a portion of the well-established statistical and metabolic association observed between these lipid and lipoprotein risk factors.

A biologic basis for the linkage of the CETP gene with the lipid factor is supported by numerous studies. CETP activity depends on the size of the pool of TG-rich lipopro- 
teins, and may explain the association between hypertriglyceridemia and reduced HDL levels (Tall, 1986). Further, Ambrosch et al. (1998) showed that increasing TG and CETP activity levels are associated with decreasing LDL particle diameters and a preponderance of a monodispersed LDL pattern, particularly in insulin-resistant subjects . In diet studies Lagrost et al. (1999) demonstrated that CETP activity influences the size distribution of LDL and HDL particles in normolipidemic individuals, and is correlated negatively with HDL cholesterol, but positively with triglyceride concentrations. Guerin et al. (1994) showed that the capacity of LDL particles to accept the cholesterol ester from HDL was highly correlated with the LDL-TG content. Finally, an in vitro study suggested that the plasma TG-rich lipoprotein level and the extent of intraplasma LCAT, CETP, lipoprotein lipase and likely HL reactions in vivo may play a role in determining the LDL phenotype (Chung et al., 1998). Thus, involvement of the CETP gene in the covariation of LDL-PPD, TG and HDL is plausible.

In this study borderline evidence for linkage was also observed between the apoE gene and the body weight/fat factor. This result is consistent with previous association studies. For example, in a study based on the Bogalusa Heart Study apoE phenotypes were significantly associated with BMI, percent body fat and plasma insulin levels, with the apo $\varepsilon$ group having significantly lower values of BMI, body fat, and plasma insulin levels than the $\varepsilon 3$ and $\varepsilon 4$ groups (Srinivasan et al., 1996). Another study suggested that the $\varepsilon 4$ phenotype significantly modifies the central obesity-induced changes in metabolic and hemodynamic variables characteristic of insulin resistance (Uusitupa et al., 1996). In the current study individuals with an apo $\varepsilon 2$ allele had lower weight/fat factor scores than individuals with either $\varepsilon 3$ or $\varepsilon 4$, however, the result was not statistically significant. If confirmed, these results in healthy women could indicate that genes involved in lipid metabolism are also involved in determining differences in body fat/obesity and insulin resistance, suggesting multilocus determination of factors contributing to the IRS.

Suggestive evidence for linkage between the lipid factor and both the apoB and HSL genes was found. Although these findings are consistent with previous reports of genetic linkage with the individual lipids and lipoproteins characterizing this multivariate factor (Austin et al., 1998; Houlston et al., 1991; Rotter et al., 1996), as well as changes in levels (Friedlander et al., 2000), the evidence for linkage is weak and should be interpreted with caution. However, it is possible that the power to detect linkage to genes with more modest effects was increased when using the multivariate phenotypes. Several other groups have shown that multivariate approaches, including using a linear combination of multiple phenotypic values, can increase power to detect linkage (Allison et al., 1998; Blangero et al., 1993; Boomsma, 1996; Jiang and Zeng, 1995; Lange and Boehnke, 1983).

Although the results from this study support the presence of genetic linkage between multivariate factors and candidate genes, several important limitations should be noted. First, an alpha value of 0.01 was used to indicate sta- tistical significance. However, multiple comparisons and the finite nature of the genome could lead to false-positive results in this setting. Secondly, it is important to note that this analysis was based entirely on women, whereas previous reports included both men and women. In addition, the limited number of dizygotic women twin pairs available for analysis may have reduced the ability to detect true genetic linkage. Third, because parental information was not available, IBD was estimated from both IBS and the allele frequency for each marker, using a subroutine in the SIBPAL module of S.A.G.E. Further, because population allele frequencies were not available for many of the markers included in this study, estimates for all candidate genes were obtained from the sample of women twins. ApoE allele frequencies in this sample of women were similar to previous reports (Cauley et al., 1993; Jarvik et al., 1997; Sing and Davignon, 1985), thus allele misspecification is not likely to account for the modest linkage between the apoE gene and the weight/fat factor. Finally, a disadvantage of sib-pair linkage analysis is that environmental and behavioral covariates cannot be simultaneously incorporated into the analysis. Combined multivariate segregation and linkage analysis using full pedigree data should be used to confirm these results and to further characterize the genetic basis of these multivariate traits.

In conclusion, evidence for genetic linkage of multivariate factors of the IRS to candidate genes has been presented, contributing to the growing body of evidence suggesting multilocus determination of the IRS. This study adds additional information by providing evidence for underlying susceptibility genes, particularly the CETP gene, influencing the covariation of these interrelated risk factors. Because these multivariate factors are characterized by risk factors for type 2 diabetes and CHD, confirming these results may have important implications for understanding the genetic basis of both conditions.

\section{Acknowledgments}

The authors would like to thank Andy Louie for his important contributions to the analysis, Le Ahn Luong, Lema Haddad and Clair Turner for genotype determinations. This research was supported by National Institutes of Health grants HL-41830 and HL-46880, and was performed during Dr. Austin's tenure as an Established Investigator of the American Heart Association. Sib-pair linkage results were obtained by use of the S.A.G.E. program package, which is supported by a U.S. Public Health Service Resource Grant (1 P41 RR03655) from the National Center for Research Resources. PT is supported by the British Heart Foundation, and KE receives support from an American Diabetes Association Research Award.

\section{References}

Allayee, H., Aouizerat, B.E., Cantor, R.M., Dallinga-Thie, G.M., Krauss, R.M., Lanning, C.D., Rotter, J.I., Lusis, A.J., \& de Bruin, T.W. (1998). Families with familial combined hyperlipidemia and families enriched for coronary artery disease share genetic determinants for the atherogenic lipoprotein phenotype. American Journal of Human Genetics, 63, $577-585$. 
Allison, D.B., Thiel, B., St Jean, P., Elston, R.C., Infante, M.C., \& Schork, N.J. (1988). Multiple phenotype modeling in gene-mapping studies of quantitative traits: power advantages. American Journal of Human Genetics, 63, 1190-1201.

Ambrosch, A., Muhlen, I., Kopf, D., Augustin, W., Dierkes, J., Konig, W., Luley, C., Lehnert, H. (1998). LDL size distribution in relation to insulin sensitivity and lipoprotein pattern in young and healthy subjects. Diabetes Care, 21, 2077-2084.

Amos, C.I., \& Elston, R.C. (1989). Robust methods for the detection of genetic linkage for quantitative data from pedigrees. Genetic Epidemiology, 6, 349-360.

Austin, M.A., King, M.C., Bawol, R.D., Hulley, S.B., \& Friedman, G.D. (1987). Risk factors for coronary heart disease in adult female twins. Genetic heritability and shared environmental influences. American Journal of Epidemiology, $125,308-318$.

Austin, M.A., Talmud, P.J., Luong, L.A., Haddad, L., Day, I.N., Newman, B., Edwards, K.L., Krauss, R.M., \& Humphries, S.E. (1998). Candidate-gene studies of the atherogenic lipoprotein phenotype: a sib-pair linkage analysis of DZ women twins. American Journal of Human Genetics, 62, 406-419.

Blackwelder, W.C., \& Elston, R.C. (1982). Power and robustness of sib-pair linkage tests and extensions to large sibships. Communications in Statistics. Theory and Methods, 11, 417-484.

Blangero, J., Williams, B-S., \& Mahaney, M.C. (1993). Multivariate genetic analysis of apo AI concentration and HDL subfractions: evidence for major locus pleiotropy. Genetic Epidemiology, 10, 617-622.

Boerwinkle, E., Xiong, W.J., Fourest, E., \& Chan, L. (1989). Rapid typing of tandemly repeated hypervariable loci by the polymerase chain reaction: application to the apolipoprotein B 3' hypervariable region. Proceedings of the National Academy of Sciences of the United States of America, 86, 212-216.

Bolla, M.K., Haddad, L., Humphries, S.E., Winder, A.F., \& Day, I.N. (1995). High-throughput method for determination of apolipoprotein $\mathrm{E}$ genotypes with use of restriction digestion analysis by microplate array diagonal gel electrophoresis. Clinical Chemistry, 41, 1599-1604.

Boomsma, D.I. (1996). Using multivariate genetic modeling to detect pleiotropic quantitative trait loci. Behavior Genetics, 26, 161-166.

Bouchard, C., \& Perusse, L. (1993). Genetics of causes and manifestations of the metabolic syndrome. In G. Crepaldi, A. Tiengo A, \& E. Manzato (Eds). Diabetes, obesity and the hyperlipidemias: The plurimetabolic syndrome (pp. 67-73). Amsterdam: Elsevier Science Publishers.

Cardon, L.R., Carmelli, D., Fabsitz, R.R., \& Reed, T. (1994). Genetic and environmental correlations between obesity and body fat distribution in adult male twins. Human Biology, 66, 465-479.

Carmelli, D., Cardon, L.R., \& Fabsitz R. (1994). Clustering of hypertension, diabetes, and obesity in adult male twins: same genes or same environments? American Journal of Human Genetics, 55, 566-573.

Cauley, J.A., Eichner, J.E., Kamboh, M.I., Ferrell, R.E., \& Kuller, L.H. (1993). Apo E allele frequencies in younger (age 42-50) vs older (age 65-90) women. Genetic Epidemiology, 10, 27-34.
Chung, B.H., Segrest, J.P., \& Franklin, F. (1998). In vitro production of beta-very low density lipoproteins and small, dense low density lipoproteins in mildly hypertriglyceridemic plasma: role of activities of lecithin:cholester acyltransferase, cholesterylester transfer proteins and lipoprotein lipase. Atherosclerosis, 141, 209-225.

Comuzzie, A.G., Mahaney, M.C., Almasy, L., Dyer, T.D., \& Blangero, J. (1997). Exploiting pleiotropy to map genes for oligogenic phenotypes using extended pedigree data. Genetic Epidemiology, 14, 975-980.

DeFronzo, R.A., \& Ferrannini, E. (1991). Insulin resistance. A multifaceted syndrome responsible for NIDDM, obesity, hypertension, dyslipidemia, and atherosclerotic cardiovascular disease. Diabetes Care, 14, 173-194.

Edwards, K.L., Austin, M.A., Newman, B., Mayer, E., Krauss, R.M., \& Selby, J.V. (1994). Multivariate analysis of the insulin resistance syndrome in women. Arteriosclerosis and Thrombosis, 14, 1940-1945.

Edwards, K.L., Mahaney, M.C., Motulsky, A.G., \& Austin, M.A. (1999). Pleiotropic genetic effects on LDL size, plasma triglyceride, and HDL cholesterol in families. Arteriosclerosis, Thrombosis and Vascular Biology, 19, 2456-2464.

Ferrannini, E., Haffner, S.M., Mitchell, B.D., \& Stern, M.P. (1991). Hyperinsulinaemia: the key feature of a cardiovascular and metabolic syndrome. Diabetologia, 34, 416-422.

Friedlander, Y., Talmud, P.J., Edwards, K., Humphries, S.E., \& Austin, M.A. (2000). Sib-Pair linkage analysis of longitudinal changes in lipoprotein risk factors and lipase genes in women twins. Journal of Lipid Research, 41, 1302-1309.

Gudnason, V., Kakko, S., Nicaud, V., Savolainen, M.J., Kesaniemi, Y.A., Tahvanainen, E., \& Humphries, S. (1999). Cholesteryl ester transfer protein gene effect on CETP activity and plasma high-density lipoprotein in European populations. The EARS Group. European Journal of Clinical Investigation, 29, 116-128.

Guerin, M., Dolphin, P.J., \& Chapman, M.J. (1994). A new in vitro method for the simultaneous evaluation of cholesteryl ester exchange and mass transfer between HDL and apoBcontaining lipoprotein subspecies. Identification of preferential cholesteryl ester acceptors in human plasma. Arterioscler Thromb. 14, 199-206.

Haseman, J.K., \& Elston, R.C. (1972). The investigation of linkage between a quantitative trait and a marker locus. Behavior Genetics, 2, 3-19.

Houlston, R.S., Snowden, C., Laker, M.F., Alberti, K.G., \& Humphries, S.E. (1991). Variation in the apolipoprotein B gene and development of type 2 diabetes mellitus. Disease Markers, 9, 87-96.

Jarvik, G.P., Goode, E.L., Austin, M.A., Auwerx, J., Deeb, S., Schellenberg, G.D., \& Reed, T. (1997). Evidence that the apolipoprotein E-genotype effects on lipid levels can change with age in males: a longitudinal analysis. American Journal of Human Genetics, 61, 171-181.

Jiang, C., \& Zeng, Z.B. (1995). Multiple trait analysis of genetic mapping for quantitative trait loci. Genetics, 140, 1111-1127.

Krauss, R.M., \& Burke, D.J. (1982). Identification of multiple subclasses of plasma low density lipoproteins in normal humans. Journal of Lipid Research, 23, 97-104.

Kuivenhoven, J.A., Jukema, J.W., Zwinderman, A.H., de Knijff, P., McPherson, R., Bruschke, A.V., Lie, K.I., \& Kastelein, J.J. 
(1998). The role of a common variant of the cholesteryl ester transfer protein gene in the progression of coronary atherosclerosis. The Regression Growth Evaluation Statin Study Group [see comments]. New England Journal of Medicine, 338, 86-93.

Lagrost, L., Mensink, R.P., Guyard-Dangremont, V., Temme, E.H., Desrumaux, C., Athias, A., Hornstra, G., \& Gambert, P. (1999). Variations in serum cholesteryl ester transfer and phospholipid transfer activities in healthy women and men consuming diets enriched in lauric, palmitic or oleic acids. Atherosclerosis, 142, 395-402.

Lange, K., \& Boehnke, M. (1983). Extensions to pedigree analysis. IV. Covariance components models for multivariate traits. American Journal of Medical Genetics, 14, 513-524.

Liese, A.D., Mayer-Davis, E.J., \& Haffner, S.M. (1998). Development of the multiple metabolic syndrome: an epidemiologic perspective. Epidemiologic Reviews, 20, 157-172.

Mahaney, M.C., Blangero, J., Comuzzie, A.G., VandeBerg, J.L., Stern, M.P., \& MacCluer, J.W. (1995). Plasma HDL cholesterol, triglycerides, and adiposity. A quantitative genetic test of the conjoint trait hypothesis in the San Antonio Family Heart Study. Circulation, 92, 3240-3248.

Mitchell, B.D., Kammerer, C.M., Mahaney, M.C., Blangero, J., Comuzzie, A.G., Atwood, L.D., Haffner, S.M., Stern, M.P., \& MacCluer, J.W. (1996). Genetic analysis of the IRS. Pleiotropic effects of genes influencing insulin levels on lipoprotein and obesity measures. Arteriosclerosis, Thrombosis and Vascular Biology, 16, 281-288.

Modan, M., Halkin, H., Almog, S., Lusky, A., Eshkol, A., Shefi, M., Shitrit, A., \& Fuchs, Z. (1985). Hyperinsulinemia. A link between hypertension obesity and glucose intolerance. Journal of Clinical Investigation, 75, 809-817.

Nagele, U., Hagele, E.O., Sauer, G., Wiedemann, E., Lehmann P, Wahlefeld AW, Gruber W. (1984). Reagent for the enzymatic determination of serum total triglycerides with improved lipolytic efficiency. Journal of Clinical Chemistry and Clinical Biochemistry, 22, 165-174.

Nichols, A.V., Krauss, R.M., \& Muliner, T.A. (Eds). (1986). Nondenaturing polyacrylamide gradient gel electrophoresis. Orlando, FL: Academic Press.

Rainwater, D.L., Mitchell, B.D., Mahaney, M.C., \& Haffner, S.M. (1997). Genetic relationship between measures of HDL phenotypes and insulin concentrations. Arteriosclerosis, Thrombosis and Vascular Biology, 17, 3414-3419.

Reaven, G.M. (1998). Banting lecture. Role of insulin resistance in human disease. Diabetes, 37, 1595-1607.

Rotter, J.I., Bu, X., Cantor, R.M., Warden, C.H., Brown, J., Gray, R.J., Blanche, P.J., Krauss, R.M., Lusis, A.J. (1996). Multilocus genetic determinants of LDL particle size in coronary artery disease families. American Journal of Human Genetics, 58, 585-594.

S.A.G.E. Statistical Analysis for Genetic Epidemiology, release 2.2 (1994). Department of Epidemiology and Biostatistics, Case Western Reserve University, Cleveland.

Selby, J.V., Austin, M.A., Newman, B., Zhang, D., Quesenberry, C.P. Jr., Mayer, E.J., \& Krauss, R.M. (1993). LDL subclass phenotypes and the insulin resistance syndrome in women. Circulation, 88, 381-387.

Sing, C.F., \& Davignon, J. (1985). Role of the apolipoprotein E polymorphism in determining normal plasma lipid and lipoprotein variation. American Journal of Human Genetics, 37, 268-285.

Srinivasan, S.R., Ehnholm, C., Wattigney, W.A., Bao, W., \& Berenson, G.S. (1996). The relation of apolipoprotein E polymorphism to multiple cardiovascular risk in children: the Bogalusa Heart Study. Atherosclerosis, 123, 33-42.

Stevens, J. (1986). Applied Multivariate Statistics for the Social Sciences. Hillsdale, NJ: Lawrence Erlbaum Associates.

Tall, A.R. (1986). Plasma lipid transfer proteins. Journal of Lipid Research, 27, 361-367.

Talmud, P.J., Edwards, K.L., Turner, C.L., Newman, B., Palmen, J.M., Humphries, S.E., \& Austin, M.A. (2000). Linkage of the cholesteryl ester transfer protein (CETP) gene to LDL particle size: Use of a novel tetranucleotide repeat within the CETP promotor. Circulation, 101, 2461-2466.

Uusitupa, M.I., Karhunen, L., Rissanen, A., Franssila-Kallunki, A., Niskanen, L., Kervinen, K., \& Kesaniemi, Y.A. (1996). Apolipoprotein E phenotype modifies metabolic and hemodynamic abnormalities related to central obesity in women. American Journal of Clinical Nutrition, 64, 131-136.

Warnick, G.R., Nguyen, T., \& Albers, A.A. (1985). Comparison of improved precipitation methods for quantification of highdensity lipoprotein cholesterol. Clinical Chemistry, 31, $217-222$ 